\title{
A SYSTEMATIC REVIEW OF GUIDED IMAGERY AS AN ADJUVANT CANCER THERAPY
}

\author{
LIZ ROFFE $^{\mathrm{a}}$, KATJA SCHMIDT ${ }^{\mathrm{b}, *}$ and EDZARD ERNST ${ }^{\mathrm{b}}$ \\ ${ }^{a}$ School of Nursing and Midwifery, University of Southampton, Highfield, Southampton, S017 $1 B J, U K$ \\ ${ }^{b}$ Complementary Medicine, Peninsula Medical School, Universities of Exeter \& Plymouth, Institute of Health \& Social \\ Care, 25 Victoria Park Road, Exeter, EX2 4NT, UK
}

\begin{abstract}
SUMMARY
Aim: The aim of this paper is to summarise and critically evaluate the evidence available from controlled clinical trials regarding the use of guided imagery as a sole adjuvant therapy for cancer patients.

Methods: Electronic searches for controlled clinical trials were carried out in eight databases and two clinical trial registers. Trials that featured guided imagery as a sole adjuvant therapy were included. No language restrictions were imposed. Data were extracted and validated independently by two researchers.

Results: Six randomised clinical trials were included. Detailed results were available for four studies only. Poor reporting and heterogeneous populations, interventions and outcome measures across trials precluded statistical pooling of results. The methodological quality was on average low. Three studies reported significant differences in measures of anxiety, comfort or emotional response to chemotherapy for patients who received guided imagery over the control groups. Two studies showed no differences between guided imagery and other interventions in any of the outcome measures.

Conclusion: Guided imagery, as a sole adjuvant cancer therapy may be psycho-supportive and increase comfort. There is no compelling evidence to suggest positive effects on physical symptoms such as nausea and vomiting. The data seem sufficiently encouraging for the use of guided imagery as an adjuvant cancer therapy to merit further research. Copyright (C) 2005 John Wiley \& Sons, Ltd.
\end{abstract}

\section{INTRODUCTION}

Guided imagery has recently been identified as one of the 10 most frequently recommended complementary cancer therapies on the Internet (Schmidt and Ernst, 2004). It is a technique used to harness the power of the mind to form mental representations of objects, places or situations, which are perceived through the senses (Post-White, 2002). The term 'visualisation' is often used interchangeably with imagery. Although visual images are most commonly evoked, sounds, smells, tastes and sensory or affective feelings may also be induced.

Guided imagery refers to the use of imagination to invoke one or more of the senses. It involves the 'guiding' of an individual through experiences in

*Correspondence to: Complementary Medicine, Peninsula Medical School, Universities of Exeter and Plymouth, Institute of Health \& Social Care, 25 Victoria Park Road, Exeter, EX2 4NT, UK. E-mail: katja.schmidt@pms.ac.uk the mind, in order to access physical, emotional and spiritual dimensions to affect bodily change (Achterberg, 1985). In a guided imagery session a practitioner or other individual leads the participant through an imagery technique or script. This can take place in group or in one-toone sessions. Recordings of spoken scripts on audiotapes are also commonly used, allowing an individual to practise in a location of their choosing. Individuals may also use imagery without the guidance of a script. Gentle background music often accompanies imagery sessions to help maintain a relaxed state and to free the mind from other thoughts.

Imagery techniques are frequently used to bring calmness and a sense of space to alleviate anxiety and pain (Spiegel, 1993; Lang and Patt, 1994) and are claimed to encourage the receptivity of treatment and facilitate the process of recovery (Simonton et al., 1978). Guided imagery techniques encourage people to feel a connection between their mind and their body and can aid 
in facilitating feelings of empowerment in order to help individuals manage certain difficulties in their lives. Studies have suggested that guided imagery may influence physiological outcomes such as white blood cell count in medical patients including cancer patients, (Donaldson, 2000), narcotic medication requirements (Tusek et al., 1997a,b) and other immunological effects (Gruber et al., 1993; Walker et al., 1997). Psycho-neuroimmunological theories propose that the psychological response to guided imagery may down-regulate the hypothalamic-pituitary-adrenal-axis, resulting in a reduced stress response, increased immune function and sense of well-being (Post-White, 1998). However, immune responses to emotional stress are complex and heterogeneous (Post-White, 2002).

Guided imagery techniques vary, but generally involve guiding the imagination towards places (environment or situation) in which the patient feels calm, safe, content, happy and relaxed. Similar to meditation, guided imagery encourages individuals to free their mind of interfering thoughts, release their concerns from daily life and become absorbed in the session (Post-White, 1998). Scripts have been developed to address particular conditions or problems, which may introduce the participant to new experiences and enable them to break out of inflexible or negative thought patterns (e.g. Post-White, 2002).

Guided imagery interventions in oncology have focused on four areas: efficacy in pain management, influence on surgical outcomes, improvement in quality of life and changes in immunity (Lee, 1999). Specific techniques for cancer patients may involve directing their thoughts to locations of the tumour or metastases. The images evoked may vary according to the technique or the individual's preference, from visualising a healing light shining on the tumour or affected area of the body, to the immune system or cancer treatment attacking and destroying cancer cells. Scripts may address concerns over treatments, provide positive thoughts and encourage new coping behaviours for managing pain, anxiety and nausea (PostWhite, 2002).

The boundaries between the different types of mind body therapies are not easily defined, and therapies are therefore often discussed collectively (Astin et al., 2003). Guided imagery is often used to aid hypnosis, or meditation, or combined with other techniques such as progressive muscle relaxation, but the technique can also be used alone. Relaxation is not always thought to be necessary for guided imagery (Post-White, 2002) although many relaxation or guided imagery sessions incorporate elements of each practice and use similar techniques. Previous reviews of guided imagery trials have included combined interventions (Wallace, 1997; Luebert et al., 2001) but did not include all randomised-controlled trials of guided imagery with cancer patients available to date. We would argue that the therapeutic effects of guided imagery as a sole adjuvant intervention should be evaluated in order to clarify its role in other therapeutic regimens.

A systematic review can be viewed as a scientific and systematic examination of the available evidence in a specific topic (Bigby and Williams, 2003). The aim of a systematic review is to systematically and thoroughly assess the best possible scientific evidence about the effects of a healthcare intervention (Cochrane Library, 2004). The method of this systematic review was based on Cochrane Collaboration principles. Our aim was to summarise and critically evaluate the evidence from clinical trials for or against the use of guided imagery as a sole adjuvant intervention with cancer patients regarding any physical or psychological change. We asked the following research questions:

Are there benefits to cancer patients from the use of guided imagery as a sole adjuvant therapy? What does the evidence from controlled clinical trials suggest regarding the effectiveness of imagery as an adjuvant cancer therapy? What outcome measures have been tested and are they appropriate? What is the methodological quality of the studies?

\section{METHOD}

Systematic literature searches were performed to identify all randomised controlled clinical trials in which an imagery intervention was applied to cancer patients. The following electronic databases were searched from their inception until March 2004: the Allied and Complementary Medicine Database (inception 1985), the British Nursing Index (1994), CancerLit, CINAHL (1983), Current Controlled Clinical Trials Register (inception 1998), DH-DATA (1983), EMBASE (1966), ISI Web of Science (inception 1975), MEDLINE (1966), National Research Register (inception 
2000) and PSYCHINFO (1987). The search terms used were cancer or oncolog\$ or carcinogen $\$$ or tumo $\$ r$ and imagery or visualisation or visualisation. Reference lists of reviews and our departmental files were hand-searched for additional trials.

Studies were required to include a control group. No restrictions were placed on the language, place or year of publication. The terminology used in the primary reports was used to determine and distinguish between the types of therapies. Articles were included if they specified the use of guided imagery, imagery or visualisation with cancer patients as a sole adjuvant intervention to standard or palliative cancer care or as a sole supportive intervention in cancer patients who were not concurrently undergoing treatment. There were no restrictions placed on the site or stage of cancer. Studies in which the guided imagery intervention was described as occurring in a support group environment, or as guided imagery combined with any other therapy or coping package, such as hypnosis, relaxation therapy, music therapy, cognitive behavioural or any other coping package were excluded.

Data were extracted independently from each paper by two reviewers (L.R., K.S.) and verified by a third reviewer (E.E.) according to pre-defined criteria. The methodological reporting of the studies was assessed using the Jadad score (Jadad et al., 1996). The Jadad score was calculated by assessing three criteria: blinding and description of the method of blinding, randomisation and description of the method of randomisation and reporting of dropouts and/or withdrawals. The maximum number of points that can be achieved on the Jadad score is 5. In guided imagery it is impossible to blind patients, as it will be obvious whether an intervention was received or not. However, one point was given for blinding if the outcome assessor was blinded. The validity of each trial was assessed according to a previously published score ranging from 0 (minimum validity) to 3 (maximum validity) where one point was given for a positive answer to each one of the following questions: (1) Was the study sample relevant? (2) Was the intervention appropriate? (3) Was the outcome measure suitable? (Ernst, 2002). The heterogeneity of populations, interventions and outcome measures across trials precluded statistical pooling of results. Results were therefore tabulated and described narratively.

\section{RESULTS}

The literature searches located 103 articles investigating guided imagery for cancer patients. From these, 97 studies did not meet the inclusion criteria. The remaining six trials were included in the review. Figure 1 summarises the exclusion process. Examples of some of the more relevant but nevertheless excluded trials are six RCTs that assessed the effects of guided imagery in combination with relaxation techniques in cancer patients (Burish et al., 1991; Gruber et al., 1993; Arathuzik, 1994; Richardson et al., 1997; Walker et al., 1999; Xie-Zhong, 2001). Five RCTs combined guided imagery with progressive muscle relaxation in the experimental group (Burish and Lyles, 1981; Lyles et al., 1982; Syrjala et al., 1995; Liu et al., 2001; Baider et al., 2001). Two RCTs combined guided imagery with music therapy (Xie-Zhong, 2001; Burns, 2002). One other RCT combined guided imagery with hypnosis or meditation in the experimental group (Rapkin et al., 1991; Targ and Levine, 2002).

Furthermore, four ongoing or completed RCTs in the UK were located from the National Research Register (http://www.update-software. $\mathrm{com} /$ national/), of which the majority are combined interventions. These assess the efficacy of guided imagery in cancer patients undergoing surgery for colorectal cancer (completed project R Molloy, Department of Surgery, Gartnavel General Hospital, Glasgow; http://www.updatesoftware.com/national/); evaluate the benefits of guided imagery and relaxation in cancer patients through a comparison of immune-targeted and non-specific imagery (ongoing project, M Spencer, Consultant in General Adult Psychiatry, Rydon House, Taunton (http://www.update-software.com/national/); assess the Psycho-neuroimmunological effects of relaxation and guided imagery alone and in combination with other interventions in patients with colorectal cancer (ongoing project, P Mack and L Walker, Institute of Rehabilitation, Hull; http://www.update-software.com/national/) and assess Quality of Life and psycho-neuroimmunological effects of reflexology, relaxation and guided imagery in patients with lung cancer (Lesley Walker).

Only four of the included studies were published in their entirety. Two studies were published as abstracts only (Post-White, 1996; Kwekkeboom, 1999). The authors were contacted but due to unpublished material the results from these studies 


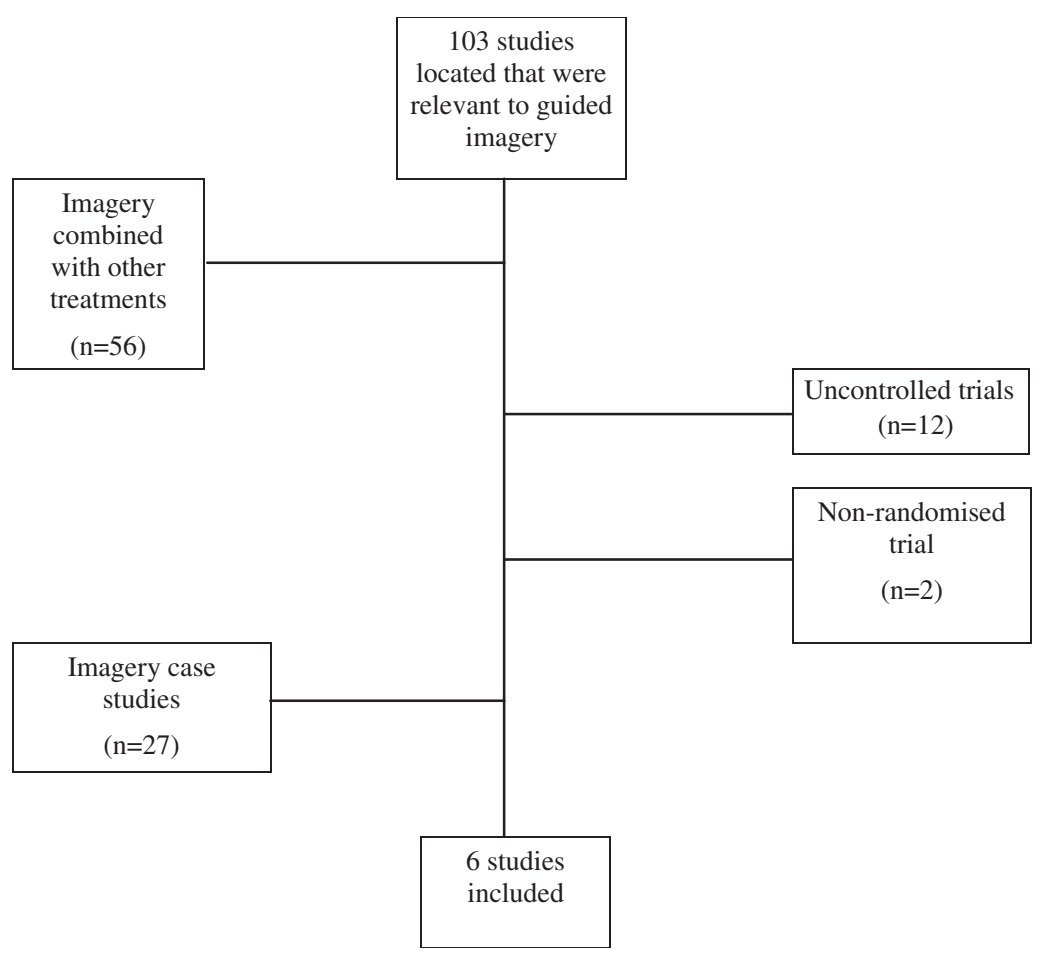

Figure 1. Flowchart of excluded studies including cancer patients.

were not available and could therefore not be evaluated. These studies are discussed narratively only. The extracted data from all included trials are presented in Table 1.

Five included studies were carried out in the USA between 1990 and 1999 and one was undertaken in Israel in 2002. All six studies were randomised controlled clinical trials, but none were outcome assessor blinded. All six trials adopted a parallel group design. In all trials patients in the experimental group received an imagery intervention additional to standard cancer care or palliative care. Patients in the control groups received standard care only, except in one study (Sloman, 2002), in which all patients received palliative care and the control condition was an 'attention' control where a nurse or practitioner spent an equivalent amount of time with patients but provided no specific therapeutic intervention. Three studies compared the guided imagery intervention with a standard care control only (Troesch et al., 1993; Kolcaba and Fox, 1999; Kwekkeboom, 1999), whereas three studies included other intervention groups such as pro- gressive muscle relaxation, hypnosis, support or a combination of guided imagery and one of the above named interventions (Feldman and Salzberg, 1990; Post-White, 1996; Sloman, 2002).

Sample sizes ranged from 30 to 75 cancer patients. Dropouts and withdrawals were mentioned in three studies (Troesch et al., 1993; Kolcaba and Fox, 1999; Kwekkeboom, 1999). All participants had previously been diagnosed with cancer and cancer types included newly diagnosed cancer, advanced primary or metastatic cancer, and early stage I or II breast cancer (Table 1). The ages of participants were reported in three studies (Troesch et al., 1993; Kolcaba and Fox, 1999; Sloman, 2002) and varied across the studies from 27 to 81 years. Gender was specified in all but one study (Troesch et al., 1993); two studies included both males and females, and in three studies participants were all female.

A variety of self-report measures were used to assess the effect of guided imagery, including anxiety, depression, quality of life, comfort, pain, nausea and vomiting, and overall chemotherapy experience. These outcomes were assessed using 


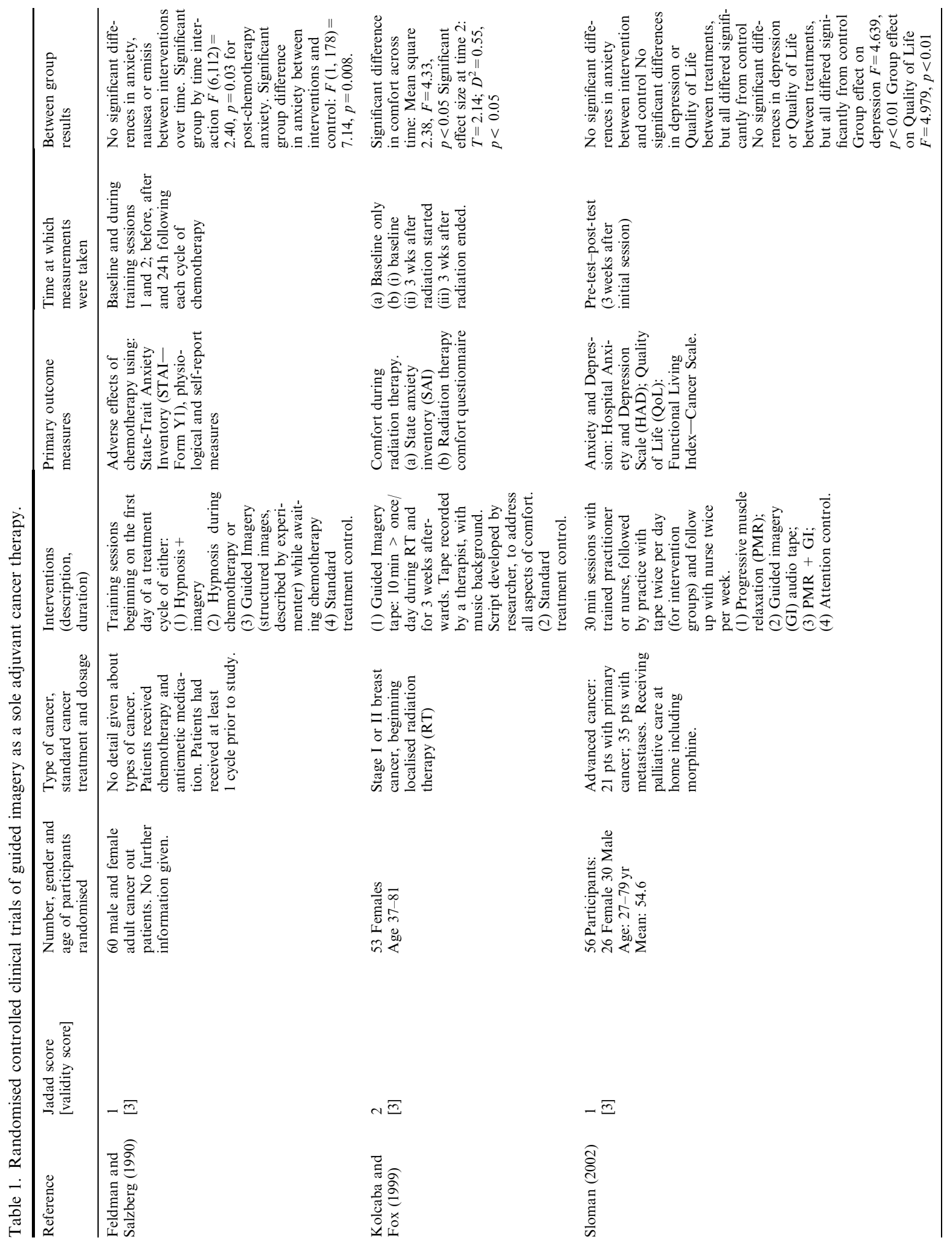




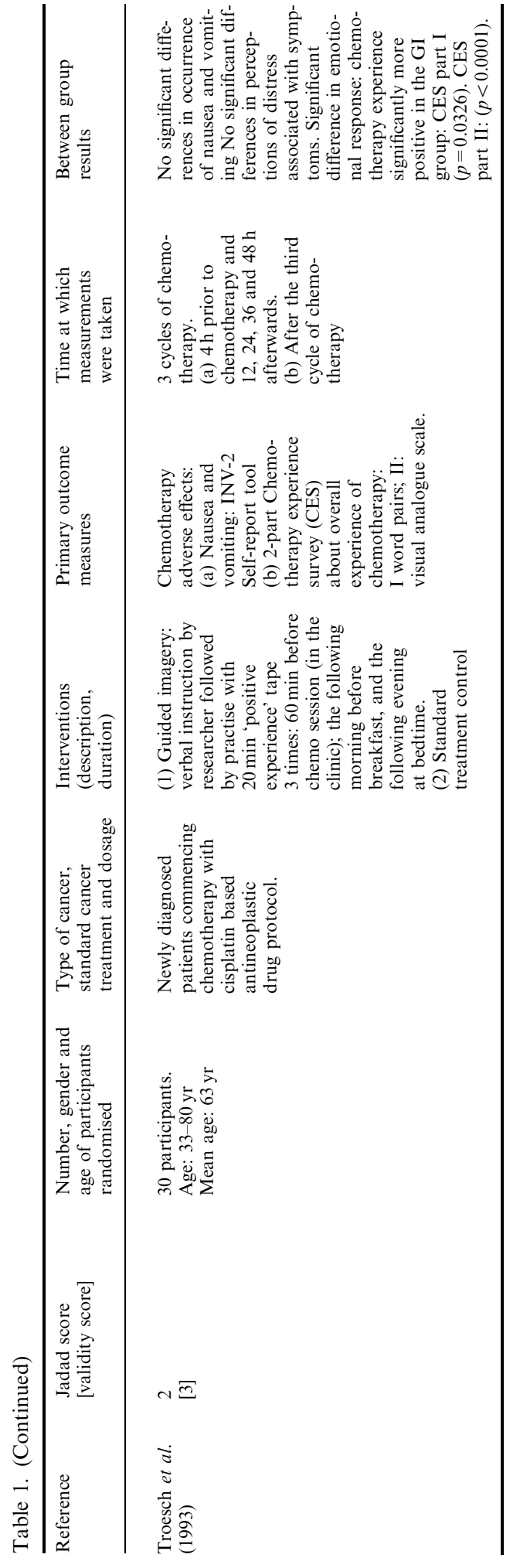

validated questionnaires, such as the State Trait Anxiety Inventory (STAI), the Hospital Anxiety and Depression Scale (HADS), the Functional Living Index Cancer Scale (FLICS), Post-chemotherapy State Anxiety Scale (PCSTAS), and the Rhodes Index of Nausea and Vomiting Form 2, and non-validated tools such as the Chemotherapy Experience survey, and the Radiation Therapy Comfort questionnaire. One abstract only study additionally measured immune function (PostWhite, 1996). Two studies investigated physiological outcomes such as heart rate and occurrence of nausea and vomiting (Feldman and Salzberg, 1990; Troesch et al., 1993). In three studies, patients were undergoing chemo- or radiotherapy; in one study patients were undergoing surgery and in another trial patients were receiving palliative care but had no other intervention. Additional cancer treatments included pain medication such as morphine and other antiemetic medication. In one study, published as an abstract only, patients had completed treatment; thus, imagery was strictly a supportive rather than an adjuvant therapy. This study was included as it met all other inclusion criteria but results were not available, and therefore it was not included in the evaluation (Post-White, 1996). All four studies published in full state that guided imagery training sessions were held either once or up to three times during duration of the trial, and duration of sessions varied from 12 to $60 \mathrm{~min}$ (Feldman and Salzberg, 1990; Troesch et al., 1993; Post-White, 1996; Sloman, 2002). No adverse effects of guided imagery were reported in any of the studies. The included studies are narratively described in the following section.

\section{SUMMARIES OF INCLUDED STUDIES}

Feldman and Salzberg assessed the effects of guided imagery on adverse reactions to cancer therapy in a randomised clinical study (Feldman and Salzberg, 1990). Sixty cancer outpatients who had recently received at least one cycle of chemotherapy were randomised into one of the following groups (a) guided imagery, (b) hypnosis, (c) hypnosis-imagery or (d) standard care. The intervention groups received training sessions, in which an experimenter briefly introduced the intervention before inducing a trance by describing 
structured images before administering chemotherapy. Measurements were taken before, after and $24 \mathrm{~h}$ following each administration of chemotherapy using the STAI and self-reports of anxiety, nausea and emesis. Results showed a significant group by time interaction for all groups for the post-chemotherapy STAI, $F(6,112)=2.40$, $P=0.03$. Additionally, significant group differences between the three intervention groups and the control group for the STAI were detected, $F(1,78)=7.14, P=0.008$. No significant differences were shown between the three different interventions or between the control group and hypnosis-imagery group, the guided imagery group or the hypnosis group alone. This study achieved one out of five Jadad points. No blinding of the outcome assessor or method of randomisation was reported.

Kolcaba and Fox studied the effects of GI on comfort in a randomised clinical study of 53 women with early stage breast cancer who were about to undergo radiation therapy (Kolcaba and Fox, 1999). The State Anxiety Inventory was used as a baseline measure of anxiety. The primary outcome measure was the Radiation Therapy Comfort questionnaire. This instrument together with the guided imagery script was developed for the study by the authors to address all aspects of comfort. The script included references to the machinery and physical environment involved in the treatment, and to a healing white light. Measurements were taken three times: (1) at baseline, (2) three weeks after radiation therapy commenced and (3) three weeks after radiation therapy ended. The results indicated that in both groups overall comfort increased over a 6-week time period. Significantly higher levels of comfort were reported in the guided imagery group compared to the control group, $F(1,51)=4.33$, $p<0.05$ over the three measurement points. The strongest effect occurred at measuring three weeks after radiation therapy commenced $\left(D^{2}=0.55\right.$, $p<0.05$ ). This study received two points on the Jadad score for methodological quality of the study. No blinding of the outcome assessor was reported.

Kwekkeboom carried out a randomised clinical trial to assess the role of imaging ability in the use of GI for cancer related pain (Kwekkeboom, 1999). Seventy-five women undergoing surgery for breast or gynaecologic cancers were randomised to receive either a GI intervention additionally to standard cancer care or only standard care control. The standard care control also allowed the use of analgesic medication. The GI intervention consisted of a 12-min relaxation tape with nature and relaxation images. Pain severity, distress and interference were the primary outcome measures. No significant differences were reported regarding the therapeutic effect of GI. Full results for this study were not available, as it was published only as dissertation abstract. Therefore, results could not be evaluated and the Jadad scoring system could not be applied to this paper.

Post-White et al. investigated the psycho-immune response to imagery and support in 73 female breast cancer survivors who had completed treatment for breast cancer within the past two years (Post-White, 1996). Participants were randomly assigned to an imagery group ('guided' imagery not specified), a support group or a standard care control group. Intervention groups met weekly for one and a half hours over a period of 8 weeks. Emotional state, fatigue, quality of life and immune responses were measured, however, no statistical analysis was reported. Again, this study is currently only available as a dissertation abstract. Therefore, the results could not be evaluated and the Jadad scoring system could not be applied to this paper.

In a randomised clinical trial, Sloman investigated the effect of guided imagery on quality of life, anxiety and depression in patients with advanced cancer (Sloman, 2002). All 56 participants were receiving palliative care in their homes and were on pain medication. Participants were randomised into four groups (a) guided imagery, (b) progressive muscle relaxation (PMR), (c) PMR and guided imagery or (d) attention control whereby nurses endeavoured giving patients an equal amount of attention as patients in the intervention group. Participants initially received a 30 min session with a trained practitioner or nurse, followed by practice with an audiotape twice per day (for the intervention groups) and follow up sessions with the practitioner twice per week. Pre-test versus post-test comparisons were carried out three weeks after the initial session with the practitioner. Outcome measures included the Hospital Anxiety and Depression Scale, and the Functional Living Index-Cancer Scale. There were no significant differences in the anxiety scores between any of the groups. Comparisons of mean depression and quality of life scores showed that none of the three treatment groups significantly differed from each other but that each treatment 
group was significantly different from the control group for both depression and quality of life (no statistics available). A significant overall group effect of the three interventions was reported for depression $(F=4.639, p<0.01)$ and quality of life $(F=4.979, p<0.01)$. The methodological quality of the study achieved one point on the Jadad score. No blinding of the outcome assessor or method of randomisation was reported.

Troesch et al. investigated the influence of GI on chemotherapy-related nausea and vomiting in a randomised clinical trial of 28 newly diagnosed cancer patients receiving the antineoplastic agent Cisplatin as a part of their chemotherapeutic regimen (Troesch et al., 1993). Patients were randomised into the experimental group, which received GI, or the control group, which received standard care alone. The experimental group was given instructions on the GI intervention from the researcher, followed by 20 -min sessions using a positive experience GI audiotape. Participants practised with the tape three times: $60 \mathrm{~min}$ before their chemotherapy session in the clinic, at home the following morning before breakfast and in the evening of the same day at bedtime. The study investigators assessed nausea and vomiting with the Rhodes Index of Nausea and Vomiting Form 2, which measures patients' perceived duration, frequency and distress of dry heaves. Data from hospital records were collected using the Subject Demographics and Emetic Response Tool. This included nurses' documentation of the frequency and severity of nausea and vomiting. However, complete results were not reported. Findings showed no statistically significant differences between the groups at any of the five measured times during chemotherapy administration. The Chemotherapy Experience survey was used to evaluate participants' overall perceptions of the chemotherapy. The GI group expressed a significantly more positive experience with chemotherapy compared to the control group $(P=0.0326)$. Two points were given to this study on the Jadad scale. No blinding of the outcome assessor was reported.

\section{DISCUSSION}

Results from both the two-armed trials showed benefits in the guided imagery intervention groups compared to standard care control groups; significant effects were found in emotional response to chemotherapy (Troesch et al., 1993) and comfort during radiotherapy (Kolcaba and Fox, 1999). Significant effects were also reported for all interventions in both the four-armed trials, in which treatment groups included hypnosis or relaxation techniques and combined interventions in addition to guided imagery alone (Feldman and Salzberg, 1990; Sloman, 2002). These studies showed positive group effects for all intervention groups on depression, quality of life and anxiety over a control or attention control group, but there were no significant differences between interventions. One four-armed study also reported significant differences between each intervention, and the control in both depression and quality of life, but no statistics were reported (Sloman, 2002). Guided imagery did not have a significant effect on physical symptoms, such as nausea or vomiting. A low incidence of symptoms at baseline may provide a reason for this in one study (Feldman and Salzberg, 1990).

Collectively, these data suggest that guided imagery may be beneficial as a psycho-supportive adjuvant therapy for cancer patients. Several caveats, however, apply. Certain aspects of reporting were inadequate in all trials. The often-low scores on the Jadad scale present a possible tendency toward bias. Details of the subject matter in the guided imagery scripts were only reported in three studies (Troesch et al., 1993; Kolcaba and Fox, 1999; Kwekkeboom, 1999) and, with the exception of one study (Kolcaba and Fox, 1999), explicit descriptions of the intervention procedures and duration were lacking. The often poor reporting of the studies available for review renders firm conclusions problematic.

The stages at which patients practised the interventions in their treatment regimen varied, as did the amount of time spent practising and the timing of measurements. Two studies reported time effects, for post chemotherapy anxiety and for comfort during radiotherapy (Feldman and Salzberg, 1990; Kolcaba and Fox, 1999). A recent meta-analysis suggested that the effect size of guided imagery increased over the first five to seven weeks but decreased at 18 weeks. (Van Kuiken, 2004). The authors also found that more detailed reporting of imagery practise and outcome measures is needed in further trials.

Most studies presented here incorporated the use of audiotapes with guidance by a nurse, practitioner or experimenter The use of audiotapes 
is often favoured over face-to-face sessions with a practitioner in order to reduced costs. From a research perspective, this also has the benefit of reducing any context effect or influence of a personal interaction on the perceived benefit of the guided imagery technique itself. However, adherence to the intervention may be harder to achieve or assess in this situation. Only one study (Kolcaba and Fox, 1999) described monitoring patients' compliance with carrying out the interventions as required by the study. The use of a support group or attention control in one study (Sloman, 2002) is commended as an attempt to control for context effects.

There were no adverse effects reported in any of the trials, but there was a lack of reporting in all but one study (Sloman, 2002) about whether any opportunities were given for comments or follow up discussions with a practitioner. It has been noted that ambiguous sensations can be evoked during guided imagery, which people may interpret very differently (Graham, 1990). The opportunity for patients to discuss their feelings and the issues raised by guided imagery, may help people acknowledge and interpret their feelings (PostWhite, 2002). This may also influence their perceptions of the therapy session and contribute to the effectiveness of the intervention. This, again, highlights the importance of careful planning, conducting and reporting of trials.

Confounding variables such as sleep, diet and exercise together with imaging ability and expectations about the intervention may influence the results of clinical trials (Kwekkeboom et al., 1988) and measuring feelings and perceptions is fraught with difficulty. Many studies that have investigated the effectiveness of imagery lack the scientific rigour of randomised controlled trials. Physiological measures provide objective and reliable measurements, which usually satisfy rigorous scientific enquiry, however, individuals' needs and reasons for using imagery may vary greatly from one another and cannot always be reflected by such measures. The impact of an intervention on quality of life issues such as the experience of treatment, perceived relaxation or anxiety, or a sense of control can only be assessed by selfreports. It is therefore important that outcome measures employed to quantify such effects are adequately validated. A social desirability effect, whereby patients report positive responses to please the experimenter may contribute to positive results (Krosnick, 1999) particularly, when questionnaires are not validated, as in two of the included trials (Troesch et al., 1993; Kolcaba and Fox, 1999). The sensitivity of frequently used diagnostic scales such as the HADS is also uncertain when employed in different clinical populations (Love et al., 2002). The HADS has been found to be less accurate for those with progressive disease (Ibbotson et al., 1994), yet this is the population it was applied to in one study (Sloman, 2002).

There is no single standard method or script for guided imagery treatment and there is currently no evidence to suggest that one form of guided imagery is more effective than another (PostWhite, 2002). Techniques employed in the reviewed studies were, where described, varied, and the nature of the therapy allows practitioners and participants to modify their practise to suit circumstances and individual requirements. Due to the highly personalised and experiential nature of the therapy, patients are currently faced with a 'trial and error' approach to selecting a technique, and are dependent on the good judgment of a welltrained and experienced practitioner.

In the two four-armed trials, no differences were found between the results of groups who received other interventions and those who received guided imagery as a sole adjuvant intervention; nor were there differences between the GI groups and those who received hypnosis or progressive muscle relaxation, in addition to GI. These results suggest there may be no differences between guided imagery as a sole intervention than when combined with other interventions such as relaxation or hypnosis for outcomes such as anxiety, depression or quality of life.

The relatively small number of studies that have investigated the effect of guided imagery as a sole adjuvant therapy may be a reflection of practice. Boundaries between the various types of mind body therapies are blurred and combinations of techniques are frequently employed according to the practitioner and the user. However, for a clear evaluation, individual therapies, (ideally individual techniques) also need to be assessed in isolation. Given the widespread use of guided imagery and its popularity, sample sizes were small and lacked sufficient power to produce widely applicable results.

Questions regarding individual differences and the influence of imagery content on effect size and immune response, as raised by Post-White and Fitzgerald (2002) still remain to be answered. 
Further research comparing different interventions, techniques (such as 'pleasant' imagery and 'targeted' imagery) and procedures (such as the timing of practise and number of sessions) and accompanied by qualitative data may reveal richer data regarding the suitability of techniques. This will enable the practitioner to address the individual needs of cancer patients from general quality of life or to a more direct approach to dealing with, for example, treatment related anxiety or adverse effects. The use of more sensitive tools and a combination of qualitative and quantitative methods might reveal subtle but important differences experienced by cancer patients. Limitations of this review include the difficulty involved in defining 'imagery' for the inclusion/exclusion criteria; and this was doubly problematic when the reporting of imagery techniques was unclear and lacked details regarding whether an intervention was adjuvant to standard oncological treatment. Even though our search strategy was thorough, we cannot be certain to have located all relevant trials. As the number of included studies is small, missing even one or two studies could alter the conclusion of this review. Furthermore, one-third of the included studies were only available in abstract form, which prevented any evaluation of their results.

In conclusion, guided imagery as a sole adjuvant cancer therapy may be psycho-supportive and increase comfort. There is, however, no significant evidence from trials to suggest positive effects on physical symptoms such as nausea and vomiting. The paucity and low methodological quality of the primary data allow only tentative conclusions. However, the evidence available to date seems sufficiently encouraging for the use of guided imagery and merits further study.

\section{ACKNOWLEDGEMENTS}

Liz Roffe was supported by a grant from the British Medical Association. Katja Schmidt was supported by the Pilkington Family Trusts.

\section{REFERENCES}

Achterberg J. 1985. Imagery in Healing: Shamanism in Modern Medicine. Shambhala: Boston.

Arathuzik D. 1994. Effects of cognitive-behavioural strategies on pain in cancer patients. Cancer Nurs 20: 79-87.
Astin JA, Shapiro SL, Eisenberg DM, Forys MA. 2003. Mind-body medicine: State of the science, implications for practice. J Am Board Fam Pract 16: 131-147.

Baider L, Peretz T, Hadani PE, Koch U. 2001. Psychological intervention in cancer patients: A randomized study. Gen Hosp Psychiat 23: 272-277.

Bigby M, Williams H. 2003. Appraising systematic reviews and meta-analyses. $J$ Am Med Assoc 139: 795-798.

Burish TG, Lyles JN. 1981. Effectiveness of relaxation training in reducing adverse reactions to cancer chemotherapy. J Behav Med 4: 65-78.

Burish TG, Snyder SL, Jenkins RA. 1991. Preparing patients for cancer chemotherapy: Effect of coping preparation and relaxation interventions. $J$ Consult Clin Psychol 59: 518-525.

Burns DS. 2002. The effects of the Bonny method of guided imagery and music on the mood and life quality of cancer patients. $J$ Music Ther 38: $51-65$.

Cochrane Library-http://212.49.218.203/newgenMB/ WebHelpSpecific/CDSR.htm (accessed 19.08.04).

Donaldson VW. 2000. A clinical study of visualization on depressed white blood cell count in medical patients. Appl Psychophysiol Biofeedback 25: 117-128.

Ernst E. 2002. Flower remedies: A systematic review of the clinical evidence. Wiener Klinische Wochenschrift 114: 963-966.

Feldman CS, Salzberg HC. 1990. The role of imagery in the hypnotic treatment of adverse reactions to cancer therapy. J S C Med Assoc 86: 303-306.

Graham H. 1990. Time, Energy and the Psychology of Healing. Jessica Kingsley Publishers Ltd.: London.

Gruber BL, Hersh SP, Hall NR et al. 1993. Immunological responses of breast cancer patients to behavioral interventions. Biofeedback Self Regul 18: $1-22$.

Ibbotson T, Maguire P, Selby P, Priestman T, Wallace L. 1994. Screening for anxiety and depression in cancer patients: The effects of disease and treatment. Eur $J$ Cancer 30A: 37-40.

Jadad AR, Moore RA, Carroll D et al. 1996. Assessing the quality of reports of randomized clinical trials: Is blinding necessary? Control Clin Trials 17: 1-12.

Kolcaba K, Fox C. 1999. The effects of guided imagery on comfort of women with early stage breast cancer undergoing radiation therapy. Oncol Nurs Forum 26: $67-72$.

Krosnick JA. 1999. Maximising measurement quality: Principles of good questionnaire design. In Measures of Political Attitudes, Robinson JP, Shaver PR, Wrightsman LS (eds). Academic Press: New York.

Kwekkeboom KL. 1999. The role of imaging ability in successful use of guided imagery for cancer related pain. $P h D$, The University of Wisconsin-Madison.

Kwekkeboom K. 2001. Outcome expectancy and success with cognitive behavioural interventions: 
The case of guided imagery. Oncol Nurs Forum 28: 1125-1132.

Kwekkeboom K, Huseby-Moore K, Ward S. 1998. Imaging Ability and Effective Use of Guided Imagery. Res Nurs Health 21: 189-198.

Lang S, Patt RB. 1994. You Don't Have to Suffer: A Complete Guide to Relieving Cancer Pain for Patients and their Families. Oxford University Press: New York.

Lee R. 1999. Guided imagery as supportive therapy in cancer treatment. Alternative Medicine Alert 2: 61-64.

Liu Y, Lin W, Liu X, Zhang J, Erika HM, Meg M. 2001. The effect of psycho-behavioral intervention on the emotional reaction and immune function in breast cancer patients undergoing radiotherapy. Acta Psychol Sinica 33: 437-441.

Love AW, Kissane DW, Bloch S, Clarke D. 2002. Diagnostic efficiency of the hospital anxiety and depression scale in women with early breast cancer. Aust N Z J Psychiat 36: 246-250.

Luebert K, Dahme B, Hasenbring M. 2001. The effectiveness of relaxation training in reducing treatment-related symptoms and improving emotional adjustment in acute non-surgical cancer treatment: A meta-analytical review. Psycho-Oncology 10: 490-502.

Lyles JN, Burish TG, Krozely MG, Oldham RK. 1982. Efficacy of relaxation training and guided imagery in reducing the aversiveness of cancer chemotherapy. Consult Clin Psychol 50: 509-524.

National Research Register. Ongoing and Completed Projects. http://www.update-software.com/national/ (accessed 18.08.04).

Post-White J. 1996. Psychoimmune response to imagery and support in breast cancer. World Congress of Psycho-Oncology, New York, October (Published in Psycho-Oncology 5: 54).

Post-White J. 1998. The role of sense of coherence in mediating the effects of mental imagery on immune function, cancer outcome and quality of life. In Stress, Coping, and Health in Families, Hamilton et al. (eds). SAGE Publications: Thousand Oaks, CA.

Post-White J. 2002. Clinical indication for use of imagery in oncology practice. In Voice Massage, Scripts for Guided Imagery, Edwards DM (ed.). Oncology Nursing Society: Pittsburgh, PA.

Post-White J, Fitzgerald M. 2002. Imagery. In Alternative/Complementary Interventions: $A$ Guide for Nurses (2nd edn), Snyder M, Lindquist R (eds). Springer: New York.

Rapkin DA, Straubing M, Holroyd JC. 1991. Guided imagery, hypnosis and recovery from head and neck cancer surgery: An exploratory study. Int J Clin Exp Hypn 39: 215-226.
Richardson MA, Post-White J, Grimm EA, Moye LA, Singletary SE, Justice B. 1997. Coping, lifestyle attitudes and immune responses to imagery and group support after breast cancer treatment. Altern Ther Health Med 3: 62-70.

Schmidt K, Ernst E. 2004. Assessing websites on complementary and alternative medicine for cancer. Ann Oncol 15: 733-742.

Simonton OC, Matthews-Simonton S, Creighton JO. 1978. Getting Well Again. J.P. Torcher Inc.: Los Angeles.

Sloman R. 2002. Relaxation and imagery for anxiety and depression control in community patients with advanced cancer. Cancer Nurs 25: 432-435.

Spiegel D. 1993. Living Beyond Limits: New Hope and Health for Facing Life Threatening Illness. Time Books: New York.

Syrjala KL, Donaldson GW, Davis MW, Kippes ME, Carr JE. 1995. Relaxation and imagery and cognitivebehavioural training reduce pain during cancer treatment: A controlled clinical trial. Pain 63: 189-198.

Targ EF, Levine EG. 2002. The efficacy of a mind-body spirit group for women with breast cancer: A randomized controlled trial. Gen Hosp Psychiatry 24: 238-248.

Troesch LM, Rodehaver CB, Delaney EA, Yanes B. 1993. The influence of guided imagery on chemotherapy-related nausea and vomiting. Oncol Nurs Forum 20: 1179-1185.

Tusek D, Church JM, Fazio VW. 1997a. Guided imagery as a coping strategy for preoperative patients. AORN J 66: 644-649.

Tusek DL, Church JM, Strong SA, Grass JA, Fazio VW. 1997b. Guided imagery: A significant advance in the care of patients undergoing elective colorectal surgery. Dis Colon Rectum 40: 172-178.

Van Kuiken D. 2004. A meta-analysis of the effect of guided imagery practice on outcomes. J Holist Nurs 22(2): 164-179.

Walker LG, Walker MB, Simpson E. 1997. Guided imagery and relaxation can modify host defences in women receiving treatment for locally advanced breast cancer. Br J Surg 84 (Suppl 1): 31.

Walker LG, Walker MB, Ogston K et al. 1999. Psychological, clinical and pathological effects of relaxation training and guided imagery during primary chemotherapy. Br J Cancer 80: 2362-2368.

Wallace KG. 1997. Analysis of recent literature concerning relaxation and imagery interventions for cancer pain. Cancer Nursing 20: 79-87.

Xie-Zhong X, Gong W, Zhengmin Y et al. 2001. Effect of music therapy and inner image relaxation on quality of life in cancer patients receiving chemotherapy. Chinese Mental Health J 15: 176-178. 